\title{
Dispersion and Performance Properties of Carbon Nanotubes (CNTs) Based Polymer Composites: A Review
}

\author{
Bhagwan F. Jogi, Mayur Sawant, Madan Kulkarni, Prakash K. Brahmankar \\ Department of Mechanical Engineering, Dr. Babasaheb Ambedkar Technological University, \\ Lonere, India \\ Email: bfjogi@dbatu.ac.in,bfjogi@yahoo.com
}

Received August 5, 2012; revised September 23, 2012; accepted October 17, 2012

\begin{abstract}
Carbon nanotubes (CNTs) based polymer composites have variety of engineering applications (electromagnetic shielding, antistatic coatings, high-strength low-density corrosion-resistant components, lightweight energy storage and many more); due to their excellent mechanical, electrical, chemical, magnetic, etc. properties. In the polymer nanocomposites CNTs are dispersed in the polymeric matrix. However the dispersion may be uniform or may not be uniform. The biggest challenge is the effective dispersion of individual CNTs in the polymer matrices, as CNTs tends to form clusters and bundles due to strong van der Waals' forces of attraction. The aggregated structure continue until physical (Mechanical) or chemical modification (Encapsulation/surface modification) of CNTs. Few modification methods such as vigorous mixing of the polymers damages CNTs structure, and may hinder their properties. But these problems can be overcome by mechanical or chemical modification of CNTs surfaces. In the chemical modification, the modifier or the long tail surfactant may encapsulate and/or partially wrap the CNTs surfaces. In this review, recent work on CNTs based polymer nanocomposite is carried out with few modifiers/encapsulating agents. Incorporation of CNTs in polymer matrix changes the performance properties such as tensile strength, tensile modulus, elongation at break, toughness, Dynamic mechanical thermal analysis (DMTA), etc. The phase morphology of the composite materials throws light on the properties of CNTs based polymer nanocomposite. Moreover phase morphology may be directly correlated with the behavior of the material, hence reviewed here through transmission electron microscopy (TEM) and scanning electron microscopy (SEM). Furthermore review is also carried out on the non-isothermal crystallization (DSC) and rheology of CNTs polymer nanocomposite.
\end{abstract}

Keywords: Carbon Nanotubes (CNTs); Encapsulation/Surface Modification; Transmission Electron Microscopy (TEM); Mechanical Properties; Crystallization

\section{Introduction}

Carbon nanotubes (CNTs) based polymer matrices creates a class of novel materials (nanocomposites) exhibiting superior mechanical, thermal, electrical and barrier properties suitable to replace many existing materials for engineering applications. In this context, use of CNTs in polymer matrices has gained considerable attention in the scientific and industrial community due to the possibility to utilize the unique (mechanical, thermal and electrical) properties of CNTs [1-4]. CNTs based polymer nanocomposites possess high stiffness, high strength and good electrical conductivity at relatively low concentrations of CNTs [5-8].

Various studies involving on single wall carbon nanotubes (SWNTs) and multiwall carbon nanotubes (MWNTs) have shown that, CNTs can have high modulus and strength levels in the range $200-1000 \mathrm{GPa}$ and $200-900 \mathrm{MPa}$ respectively [2,4]. Other studies shows that, CNTs have unique electrical properties, capable of acting as metallic-like conductors or having characteristics of a semiconductor depending upon the distortion or "chirality" of graphite lattice $[3,9,10]$. In addition, CNTs have very large aspect ratios, as high as $100-1000$ [2]. These special properties make CNTs as excellent candidate for high strength and electrically conductive polymer nanocomposite applications. Electronics and automotive are the two major areas in which CNTs used as filler material in polymer composites. Electronic applications, particularly in the semiconductor field, CNTs are used to dissipate unwanted static charge buildup. This dissipative effect is achieved by thoroughly dispersed CNTs in a given polymer matrix, where CNTs form an interconnecting conductive pathway for charge to flow $[2,11]$. In conductive polymer composites, conductive compounds need very low fibril loading less than $2 \mathrm{wt} \%$ to achieve electrostatic dissipation, compared to $8-20 \mathrm{wt} \%$ for carbon black based compounds [6]. In the 
automotive industry, CNTs are used as a conducting agent to aid in electrostatic painting. Several researcher groups have reported the encouraging results regarding the possibility of introducing CNTs for mechanical property enhancement in polymer/CNTs composites $[11,12]$. However, the magnitude of reinforcement by CNTs is found to be limited due to the challenges involved due to the dispersion of CNTs in a molecular level or at least in the forms of "ropes" (in case of SWNTs) in polymer matrices. The problem associated with homogeneous dispersion of CNTs in polymer matrices arises primarily due to the strong van der Waals' interactions between the CNTs making the polymer chain difficult to intercalate. In this context, synthesis induced "entangled" and "aggregated structure" of CNTs magnifying the problem of homogeneous dispersion. Coupled with the issue of homogeneous dispersion of CNTs in polymer matrices, poor interfacial adhesion between CNTs and polymer is another critical parameter, which dictates the efficiency of load transfer in the CNTs based polymer composites [13].

Three methods are commonly used to incorporate CNTs into polymer composites: a) Film casting of suspensions of CNTs in dissolved polymers; b) In-situ polymerization of monomer in presence of CNTs; and c) Melt-mixing of CNTs with polymers [14]. However, these methods are only composite preparation methods. It may or may not help for the dispersion of the CNTs.

\section{State of Dispersion of CNTs in Polymer/ CNTs Composites}

The issues of CNTs agglomeration/aggregation in polymer matrix is found in most of the cases irrespective of the method of composite preparation. In this connection, it is to be pointed out that melt-mixing is the preferred method of polymer/CNTs composite preparation. The tendency to form the aggregates may be minimized by appropriate application of shear during the melt-mixing (Mechanical method). In this context, literature pointed out that several equipments such as DACA microcosmpounder, DSM microcompounder, Haake miniature laboratory mixer, Buss Kneader, and twin-screw extruder have been utilized during melt-mixing to prepare the CNTs based polymer composites. In this context, two ways of introducing CNTs in polymer matrices have been reported for melt-mixing. In the first case, CNTs are directly incorporated into the polymer matrix $[13,14]$ whereas, in other case, commercially available masterbatches of CNTs are melt-mixed with polymer matrix [15]. CNTs are diluted by the pure polymer in a subsequent melt-mixing process. Composites of Polyethylene (PE)/SWNTs, Polycarbonate (PC)/MWNTs [15], Polyamide6 (PA6)/MWNTs [14], Polyamide12 (PA12)/ SWNTs [16], PA/SWNT, PMMA/SWNT [17-22] and many more are the few examples where the melt-mixing technique has been employed. Bhattacharyya et al. [14] reported the presence of significant aggregation of SWNTs in Polypropylene (PP)/SWNTs composites containing 0.8 $\mathrm{wt} \%$ SWNTs observed under optical microscope. In the Figure 1 black spots indicate the aggregates of SWNTs in PP/SWNTs composites.

Several methods have been used to modify CNTs surfaces chemically. One approach is to attach chemical groups covalently. Unfortunately, this strategy creates defects in the CNTs lattice, which can lower the electrical and thermal conductivity of the CNTs and compromise their mechanical properties. Alternatively, non-covalent interactions can be used to either encapsulate/wrap surfactants or polymers around CNTs, or to adsorb aromatic structures to the side-walls. These surface-modification methods are milder than the covalent approach, and usually cause little disruption to the structure or conductivity of CNTs. The idea of chemical modification of CNTs is to increase the interfacial adhesion with wettability of polymer/CNTs composites and ultimately the strength and other mechanical properties of the composites. Means the surfaces of CNTs are to be modified before mixing with the polymers. But the near-perfect structures of CNTs make the process more challenging, as there are no defects upon which to anchor a pendent group, and nanotubes are chemically inert in nature $[1,23]$.

Ajayan et al. [1] have been presented three methods for modification of the CNTs as shown in Figure 2. In that a) Aromatic molecules can be appended to CNTs using certain non-covalent interactions (known as $\pi-\pi$ interactions); b) Non-covalent interactions (including $\pi-\pi$ interactions, van der Waals' forces and charge-transfer interactions) can be used to encapsulate/wrap polymers around surfaces of CNTs; c) Chemical groups can be covalently attached to CNTs. Furthermore, Bose et al. [24] modified the MWNTs with the help of various modifiers as a chemical modification process.

The solution experiments are as shown in Figure 3 depicts the effect of the modifiers. Interestingly, they

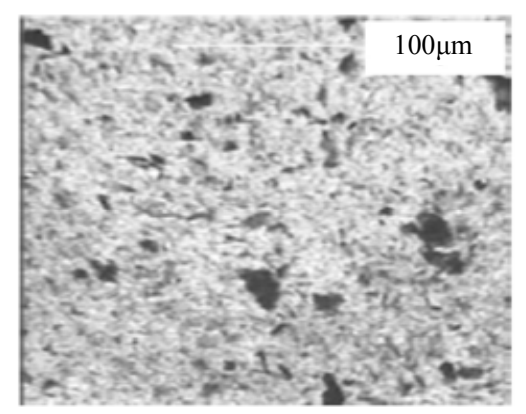

Figure 1. Optical micrograph (without cross-polars) of polypropylene/SWNT composite containing $0.8 \mathrm{wt} \%$ SWNT [14]. 


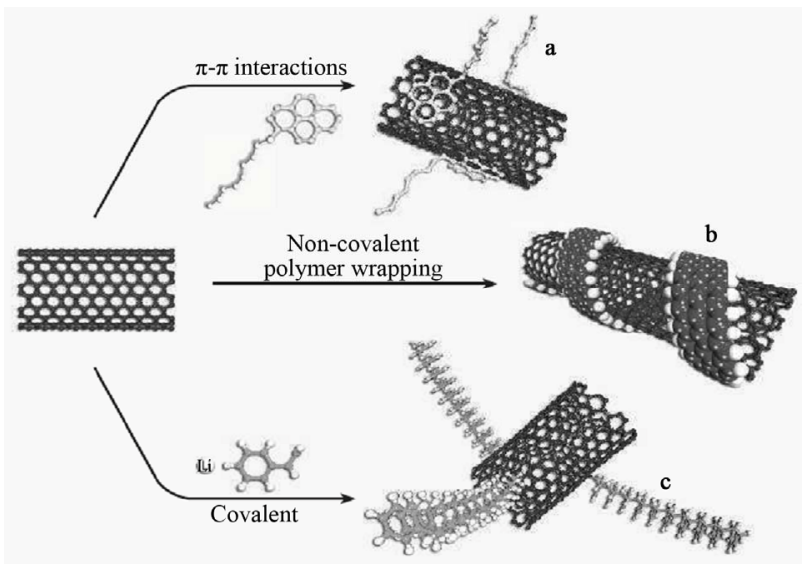

Figure 2. Chemical modification of carbon nanotubes [1].

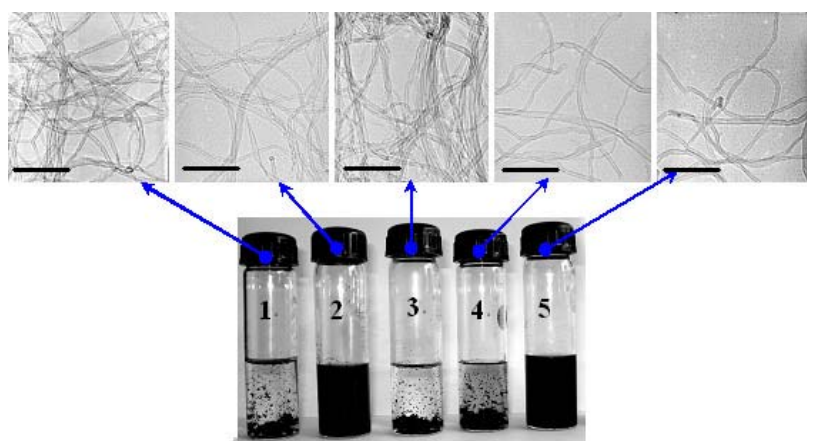

Figure 3. Dispersion of (1:1) modifier/MWNTs mixture in deionized water following sonication: (1) pure MWNTs; (2) Na-AHA modified MWNTs; (3) BTPC modified MWNTs; (4) DTPB modified MWNTs; (5) OTPB modified MWNTs. The inset shows TEM images of the respective MWNTs/ dispersant mixture; scale bar: $100 \mathrm{~nm}$ [24].

found that the Octadecyl Triphenyl Phosphonium Bromide (OTPB) modified MWNTs reveal a homogeneous dispersion and stable suspension of MWNTs. According to the comparison of modifiers, Sodium salt of 6 amino hexanoic acid (Na-AHA) modified MWNTs solution appears to be black as compared to the other phosphornium based modifiers. They found that transmission electron microscopy (TEM) images indicate a substantial exfoliation of the CNTs in case of OTPB modified MWNTs as compared to the other phosphonium based modifiers and even Na-AHA modified MWNTs.

Furthermore, during the manufacturing processes of the nanocomposites, there is no evidence of segregation to the CNTs in the extruded sample as well as no preferred alignment of CNTs observed. Figure 4 shows the TEM micrographs of polycarbonate (PC)/MWNTs composites [25]. Liu et al. [26] reported a fine and homogeneous dispersion of functionalized MWNTs (1 wt \%) in polyamide6 (PA6)/MWNTs composites. It is reported that the random dispersion of individual CNTs are seen in Figure 5, without preferred alignment or orientation after melt-mixing within the PA6 matrix, and with no evidence of CNTs aggregation. Further it is also observed that the most CNTs remain curved or even interwoven in the composites, indicating extreme flexibility of CNTs.

Apart from the direct methods (Transmission electron microscopy TEM, Scanning electron microscopy SEM, Atomic force microscopy AFM) few researchers also reported certain indirect methods to study the state of dispersion of CNTs in the polymer matrix. One of the indirect methods used to compare the state of dispersion of CNTs at a given concentration (e.g. depending on the processing conditions or addition of modifiers) is electrical conductivity measurements [11]. Sandler et al. [27] developed a dispersion process for dispersion of CNTs in an epoxy resin. The intense stirring method was chosen for dispersion of untreated catalytically grown CNTs in epoxy resin. These epoxy/CNTs composites showed conductivity around $10^{-2} \mathrm{~S} / \mathrm{m}$ with filler content as low as $0.1 \mathrm{vol} . \%$. On the other hand, characterization or quantification of the state of CNTs dispersion is a difficult task.
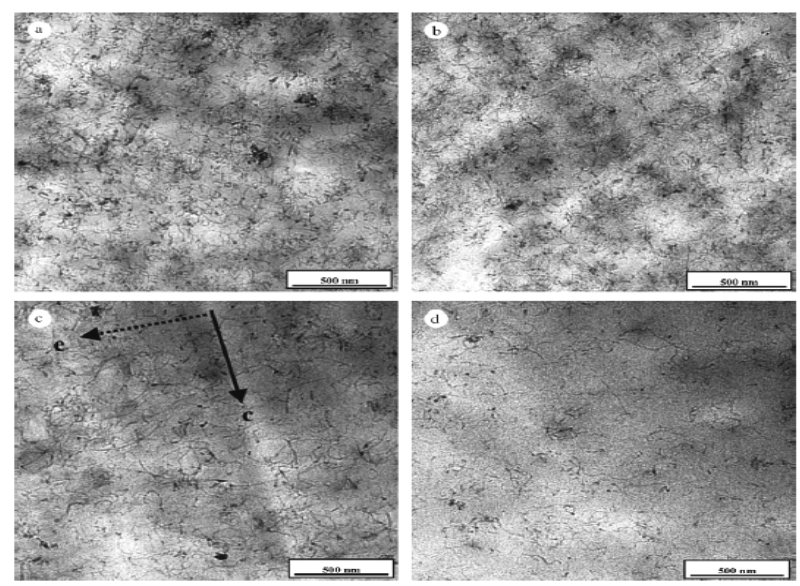

Figure 4. TEM micrographs of PC/MWNTs composites (a) 5 wt \% of MWNTs cut perpendicular to extrusion (b)-(d) cut along the extrusion direction [25].

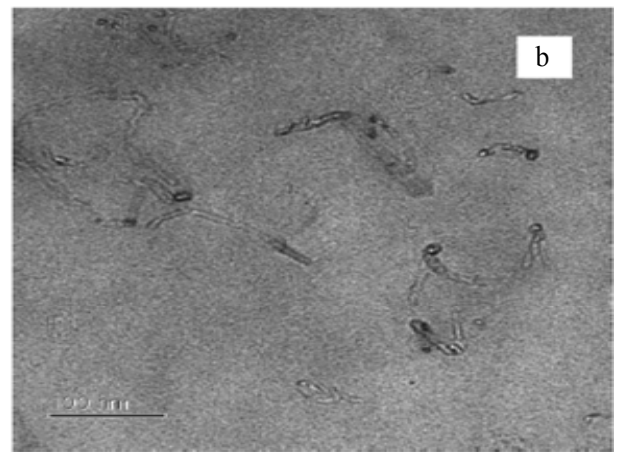

Figure 5. TEM images showing CNTs dispersion from the ultra thin section of PA6 nanocomposite containing $1 \mathrm{wt} \%$ MWNT at higher magnification [26]. 
Direct microscopic observation of CNTs dispersion in composites is difficult due to the extreme differences in radial and axial dimensions of the CNTs. Optical microscopy (OM) only assesses very big agglomerates of CNTs and is incapable to analyze the dispersion at the submicron scale. Surface based methods, such as scanning electron microscopy (SEM) and atomic force microscopy (AFM) only show a cross section of the threedimensional arrangement of the nearly one-dimensional CNTs. These problems make it difficult to observe an entire CNTs or to distinguish between different CNTs and also difficult to conclude from micrographs a threedimensional structure and state of dispersion.

Kumar et al. [28] observed the evidence of segregation in PP/nanofiber composites. Figure 6 shows the photograph of dispersion of CNTs in polymer matrix through solution method. Tetrahydrofuran (THF) is selected as a solvent for solution method where MWNTs is in wt $\% 15$, $5,4,3,2,1.5,1,0.5$ from right to left. Surface segregation is an important issue in multiphase composite systems during injection molding and subsequent materials cooling in the mold.

Safadi et al. [29] used ultrasonic energy to obtain uniform dispersion of as received MWNTs in polystyrene matrix via spin casting method. MWNTs were found to align at the shear rates generated by spin casting process. In these composites higher orientations of MWNTs near the high shear surface and more random orientation at the shear free fluid air interface was reported. Sandler et al. [27] also studied carbon nanofibers reinforced poly (ether ether ketone) PEEK/composites reported alignment of carbon nanofibers in the direction of flow during processing.

Bhattacharyya et al. [14] studied the orientation of SWNTs in Polypropylene (PP) matrix using wide angle X-ray diffraction (WAXD) and Raman spectroscopic analysis. They found that the higher orientation of SWNTs in the drawn PP/SWNTs composite fibers than pure PP. They showed (Figure 7) tangential mode Raman spectra of PP/SWNTs composite fiber with drawn ratio of 4.5. They concluded that Raman scattering intensity showed monotonically decreasing trend with in-

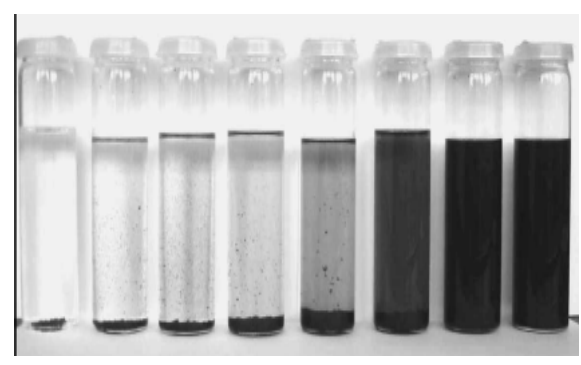

Figure 6. Photograph of composite dispersion in THF as a characterization of state of dispersion of MWNTs dispersion [28].

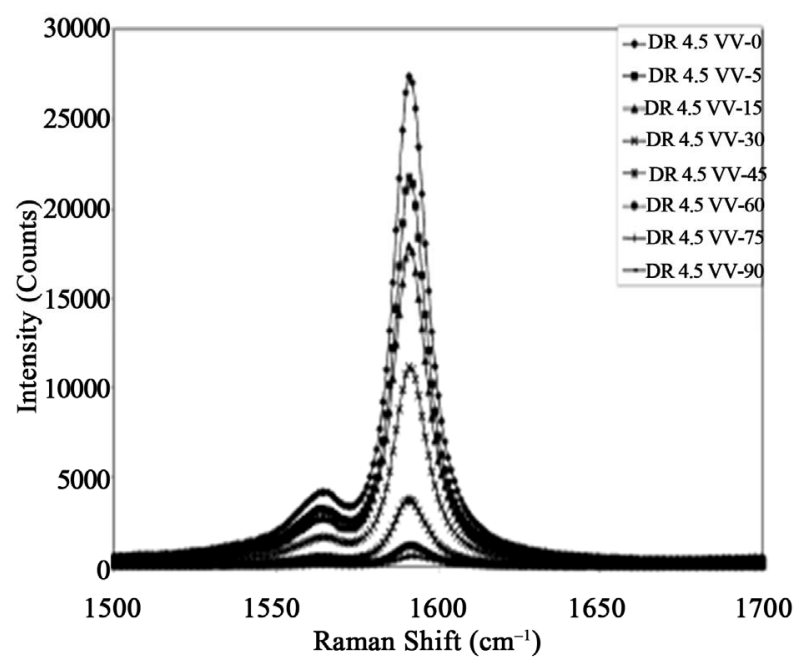

Figure 7. Tangential mode Raman spectra of PP/SWNTs composite fiber (draw ratio $=4.5)$ under VV configuration. From top to bottom, the angle between fiber axis and polarization direction of polarizer is $0,5,15,30,45,60,75$ and 90 degrees [14].

creasing the angle between the fiber axis and the polarization direction of the polarizer.

\section{Performance Properties of Polymer/CNTs Composites}

The mechanical behavior of CNTs based polymer composites are strongly decided by the contribution of each component, as well as by the phase morphology developed during mixing of CNTs and polymers (melt-mixing/compounding) and the interfacial adhesion between the phases. It is well realized that elongation at break and toughness are the important tools to monitor the adhesion between phases (CNTs/polymers) whereas the tensile strength is affected by the phase morphology, domain size and size homogeneity, etc.

However, polymer nanocomposites especially CNTs in polymer matrix changes the performance properties (tensile strength, tensile modulus, elongation at breaks toughness, etc) of the advanced new materials. Promising results were also observed while studying the composites of PA12/SWNTs reported by Bhattacharyya et al. [16]. They studied the styrene maleic anhydride (SMA) copolymer encapsulated SWNTs and the nanocomposites were prepared by melt-mixing with PA12 to obtain homogeneous dispersion of SNWTs. Furthermore they found the reactive coupling between amine end group of PA12 and maleic anhydride functionality of SMA through attenuated total reflection Fourier transform infrared (ATR-FTIR) spectroscopy. It means that better dispersion and enhanced interfacial adhesion between PA12 and SWNTs and enhancement in mechanical properties of composite material is observed. Figure 8 
depicts the representative stress-stain curve of composites of PA12/SWNTs and SMA8.

Jogi [30] prepared polymer encapsulated MWNTs using ultrasonically initiated styrene maleic anhydride (SMA) as a compatibilizers as well as modifier for MWNTs. These SMA encapsulated MWNTs were utilized to obtain melt-mixed composites of polyamide6/ acrylonitrile butadiene styrene (PA6/ABS) blends based nanocomposites. They found that polymer encapsulated MWNTs (especially with ' SMA) well dispersed in PA6 matrix and showed improvement in the storage modulus.

Meincke et al. [31] analyzed composites of polyamide6 (PA6) and CNTs. Tensile tests of the composites show a significant increase of $27 \%$ in the Young's modulus; however, the elongation at break of these materials dramatically decreases due to an embrittlement of the polyamide6. Blends of PA6 with acrylonitrile butadiene styrene (ABS) have been prepared by extrusion. CNTs filled blends show superior mechanical properties in the tensile tests. They also found that the elongation at break, as an indicator for the toughness of the materials, decreases dramatically in the presence of CNTs or carbon black (Figure 9).

The incorporation of CNTs in the polymer matrix increases the mechanical properties of final nanocomposites material. However, various parameters required to optimize to obtain the desirable behavior of the materials. The parameters may be the type of CNTs (SWNTs and MWNTs), diameter of CNTs, chirality of the CNTs and the composite preparation approach (melt-mixing/film casing) and/or the machine parameters (viz. torque, screw speed, pressure, etc.).

\subsection{Dynamic Mechanical Thermal Analysis (DMTA) of Polymer/CNTs Composites}

It has been well recognized that dynamic mechanical thermal analysis (DMTA) measurements can be em-

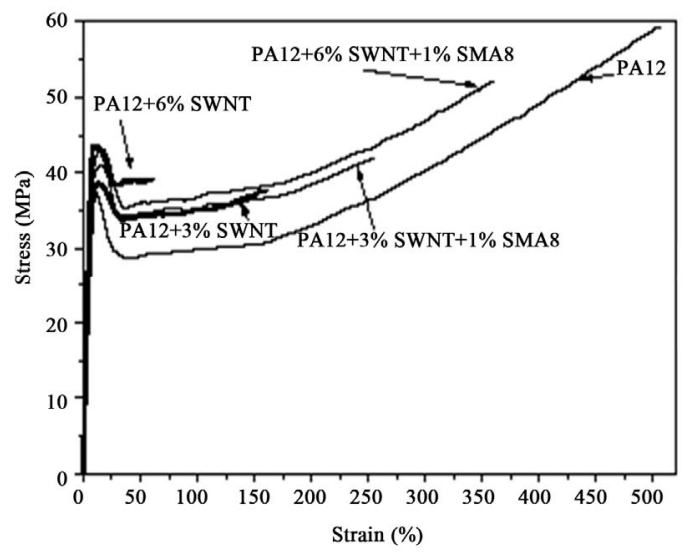

Figure 8. The representative stress-strain curve of composites of PA12/SWNTs and SMA8 [16]. ployed to get further insight into the phase transition behavior of the blends. Figures 10 and $\mathbf{1 1}$ show the variation in storage modulus $\left(E^{\prime}\right)$ and loss modulus $\left(E^{\prime \prime}\right)$ as a function of temperature for the polymer blends with MWNTs.

Jin et al. [32] analyzed the storage moduli of polymethylmethacrylate (PMMA) with varying MWNTs contain in the nanocomposites. They found an increase in the storage modulus of PMMA in the presence of the stiffening effect of the CNTs, which is particularly significant at higher temperatures. In addition, the presence of CNTs also enables the matrix to sustain a high

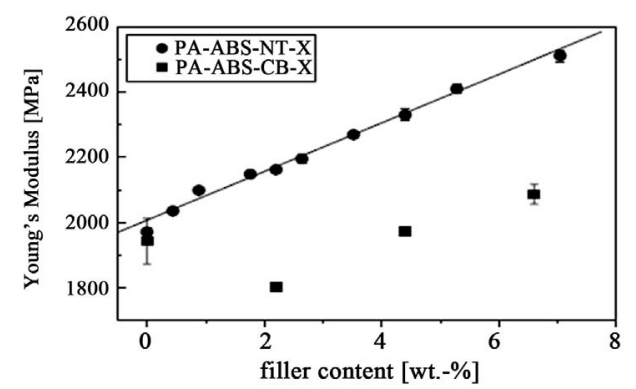

(a)

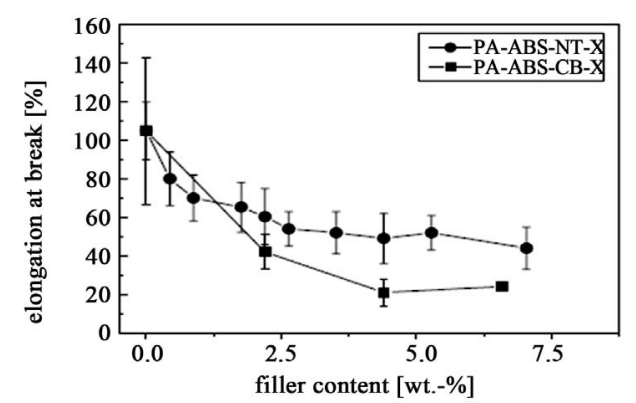

(b)

Figure 9. Young's modulus (a) and elongation at break (b) of PA6/ABS/CNTs-x samples [31].

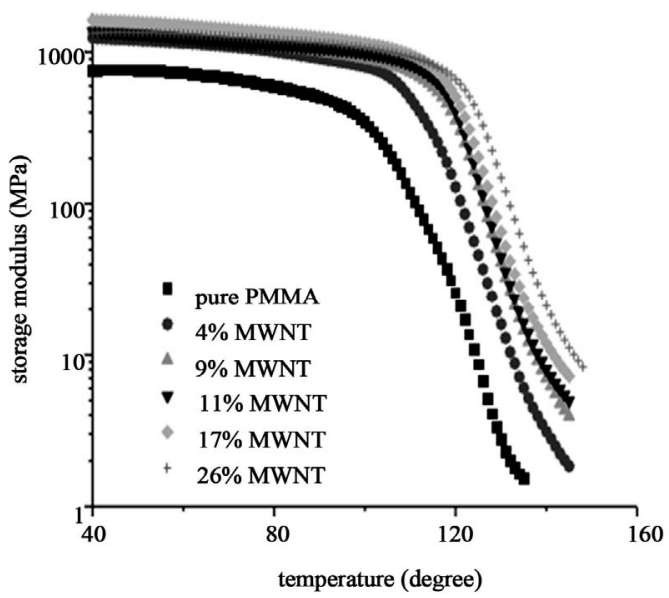

Figure 10. Storage moduli of PMMA and various composites [32]. 
modulus value to higher temperature. Figure 11 depicts the $\tan \delta$ peaks of the PMMA/MWNTs composites. The peak moves to a slightly higher temperature with increasing CNTs content, showing that the $\mathrm{T}_{\mathrm{g}}$ of the nanocomposites is raised by the addition of CNTs [32].

Bose et al. [24] discusses the variation in the storage modulus (E') and loss modulus (E") as a function of temperature for the blends of 50/50 PA6/ABS with MWNTs. They observed the higher E' at lower temperature in the presence of MWNTs based 50/50 PA6/ABS blends as compared to the individual blends constituents.

It may be due to the stiffening effect of the MWNTs. They also studied the effect of the modifier (Sodium salt 6-amino hexanoic acid (Na-AHA) modified MWNTs $1: 1,2 \mathrm{wt} \%$ ) on the dispersion of the CNTs and mechanical properties. The enhancement in E' is observed (especially in the glassy region) in the blends with as compared to the blends with p-MWNT (2 wt \%) may be due to the better dispersion of the CNTs in the presence of suitable modifier.

They concluded from Figure 12(b) that in the presence of MWNTs, $T_{\alpha}$ increases slightly as compared to 50/50 PA6/ABS blends, indicating the restriction to chain mobility due to the presence of MWNTs and ultimately increase in the mechanical properties.

\subsection{Surface Fracture Morphology and Dispersion of CNTs in Polymer Composites}

Incorporation of CNTs in polymer matrix results changes in the molecular architecture of the matrix modified to the structural changes (interface of CNTs and polymer matrix) of the nanocomposites. These structural changes can be studied using direct observation through microscopy such as SEM and TEM. Recently few researchers reported interesting findings on tensile fracture morphology of polymer CNTs composites.

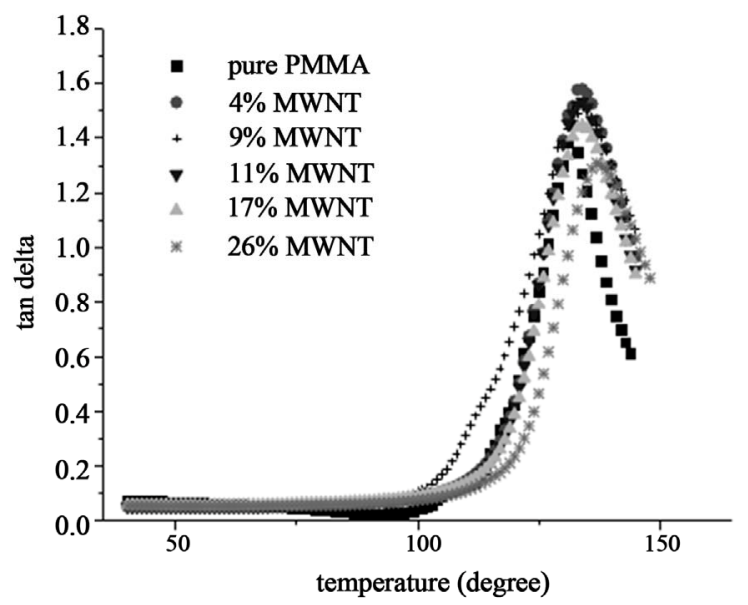

Figure 11. Tan $\delta$ peaks of PMMA and various composite [32].
Liu et al. [26] reported bead like phase morphology along stretched MWNTs and their bundles in fractured PA6/MWNTs composites observed under SEM. They found that the CNTs were not entirely pulled out but fully stretched during the tensile deformation and most of the stretched CNTs or bundles were found well aligned. Figure 13 shows SEM images of tensile fractured sur-

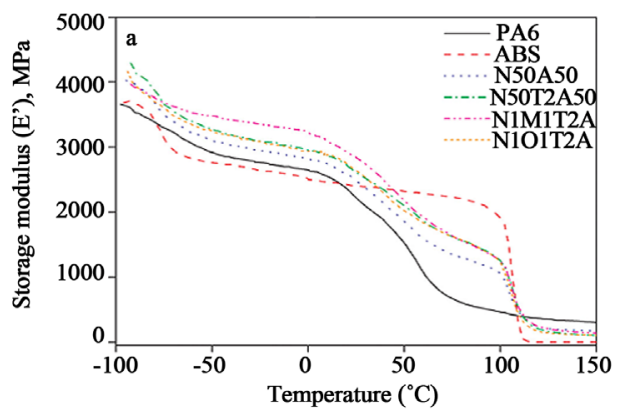

(a)

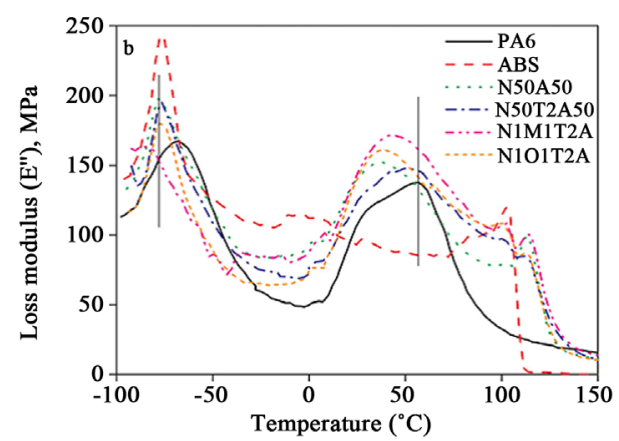

(b)

Figure 12. Dynamic mechanical thermal analysis (DMTA) of PA6/ABS blends with MWNTs for various compositions: (a) plot of storage modulus (E') versus temperature and (b) plot of loss modulus ( $E$ ”') versus temperature [24].
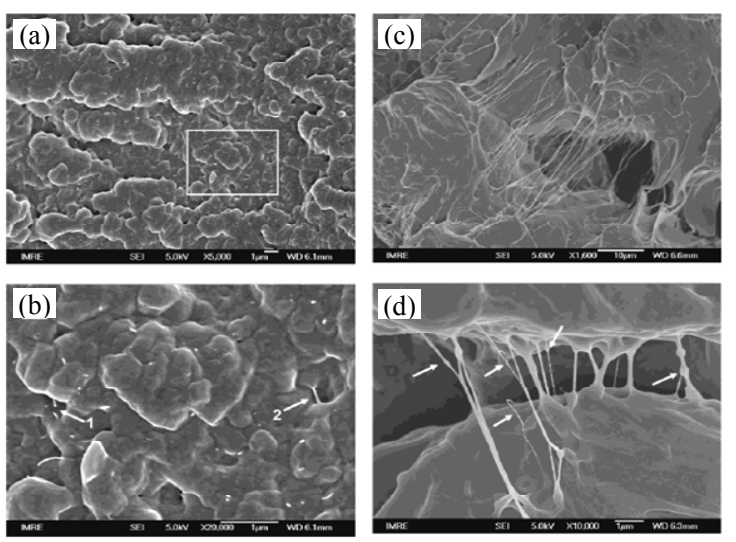

Figure 13. (a) SEM image showing overall morphology of failure surface for PA6 composites containing 0.5\% MWNTs (b) Enlarged morphology of encircled area in (a), (c) SEM of microcracks linked by stretched nanotubes and their bundles in PA6 composites (d) Enlarge of microcrack connected by MWNT sheathed with polymer in PA6 matrix [26]. 
face for PA6 composites containing $0.5 \%$ MWNTs. The issue of interfacial adhesion between polymer matrix and CNTs can be very well understood from the SEM micrographs (Figure 13) of tensile fractured surface of PA6/ MWNTs composites. It has been reported that chemically functionalized MWNTs found to be well dispersed in PA6 matrix indicating a good matrix-CNTs interface and thus a better load transfer to CNTs [26].

Kodgire et al. [33] have been analyzed the role of modifiers (sodium salt of 6-aminohexanoic acid, NaAHA) for supporting debundling the MWNTs. They found that the existence of "cation- $\pi$ " interaction between Na-AHA and MWNTs which may helps for homogeneous dispersion of CNTs within the polymer matrix during melt-mixing.

They also suggest the establishment of reactive coupling between amine functionality of Na-AHA and acid end group of PA6 during melt-mixing. The effect of the solvent and the concentration of the modifier on the dispersion of the CNTs is also studied and shown in Figure 14.

Jagatap et al. [34] studied the cryogenically fracture surfaces of MWNTs composites and are shown in Figures 15(a) and (b). The composite containing unmodified MWNTs (Figure 15(a)) clearly demonstrate the presence of agglomerated CNTs. Although homogenously dispersed bright dots which are broken end of CNTs was observed in the case of half neutralized adipic acid (HNAA)-modified MWNTs composite Figure 15(b).

This result also indicates the modification of MWNTs

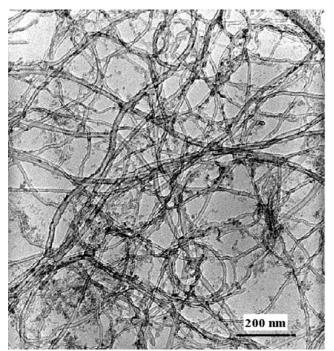

(a)

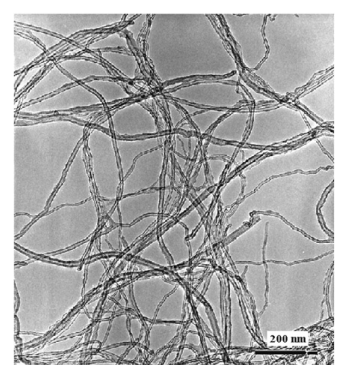

(c)

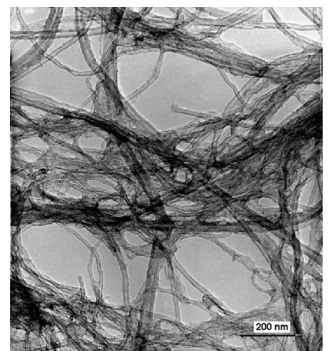

(b)

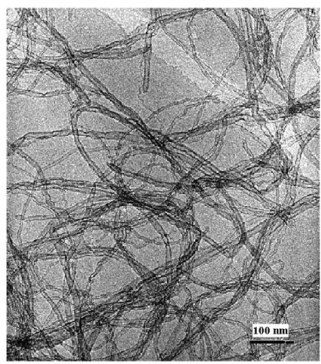

(d)
Figure 14. TEM image of (a) “aggregated” MWNTs in tetrahydrofuran (THF); (b) 1:1 mixture of MWNTs and Na-AHA in THF; (c) 1:4 mixture of MWNTs and Na-AHA in THF; (d) 1:4 mixture of MWNTs and Na-AHA in distilled water [33]. provides not only good dispersion but also strong interfacial interaction and good compatibility with polyethylene oxide (PEO). TEM images of PEO/MWNTs $(0.5 \mathrm{wt} \%)$, PEO/MWNTs $(0.5 \mathrm{wt} \%) / \mathrm{HNAA}$, and PEO/MWNTs $(1$ wt $\%$ )/HNAA are shown in Figures 16(a)-(c). The PEO/ MWNTs composite shows agglomerated nanotubes throughout the image indicating poor dispersion as observed from Figure 16(a) whereas the PEO/MWNTs $(0.5 \mathrm{wt} \%)$ HNAA composite exhibits exfoliated nanotubes (Figure 16(b)).

\subsection{Crystallization Behavior of Polymer/CNTs Composites}

It has been reported in the literature, that CNTs act as heterogeneous nucleating agent and influence the rate of crystallization and the crystallization temperature of the semi-crystalline polymer matrix.

Khare et al. [35] studied the crystallization behavior of the CNTs based polymer blend for compatibilized polypropylene (PP)/ABS blend in the presence of MWNTs. Figures 17 and 18 exhibits the crystallization exotherms of PP/ABS blend with $1 \mathrm{wt} \%$ MWNTs results in a slight increase in the crystalline temperature $\left(\mathrm{T}_{c}\right)$ of PP, suggesting the heterogeneous nucleating action of MWNTs. However, they found a significant increase in the $T_{c}$ in the case of blend with PP grafted maleic anhydride (PP-
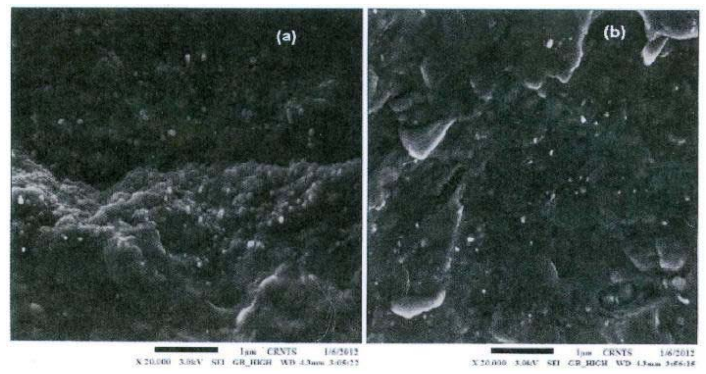

Figure 15. SEM photograph for fracture surface of (a) PEO/ MWNTs (0.5 wt\%) (b) PEO/MWNTs (0.5 wt\%)/HNAA [34].

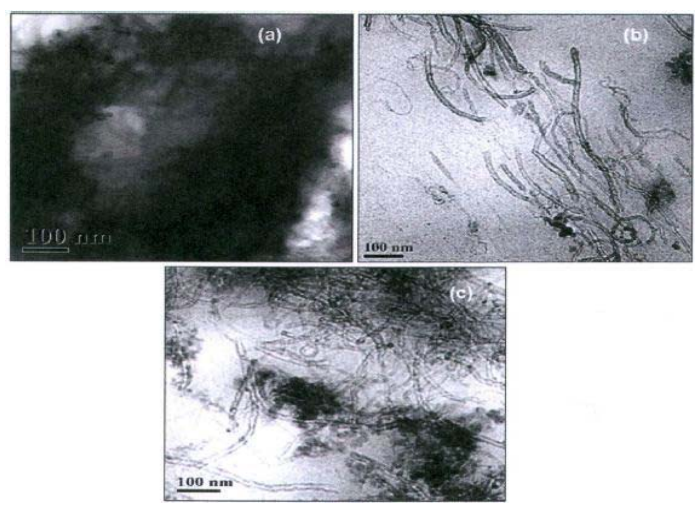

Figure 16. TEM photograph of (a) PEO/MWNTs (0.5 wt\%) (b) PEO/MWNTs (0.5 wt\%)/HNAA (c) PEO/MWNTs (1 wt \%) /HNAA [34]. 


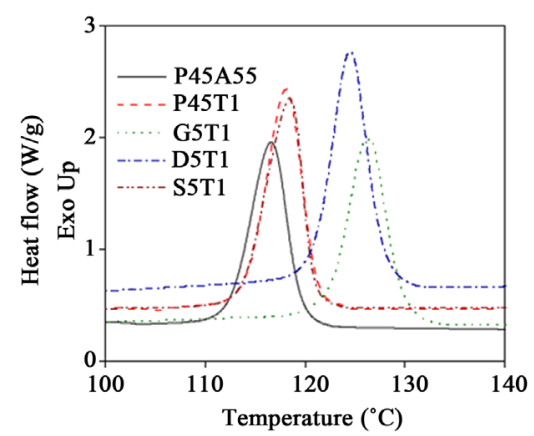

Figure 17. DSC crystallization exotherms for $45 / 55$ (wt/wt) PP/ABS blends with various compatibilizers along with MWNT [35].

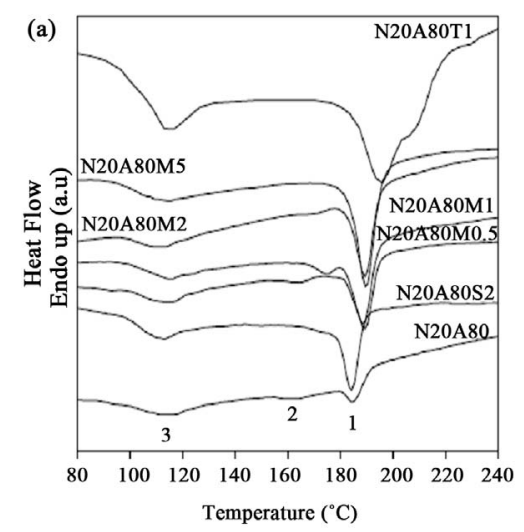

(a)

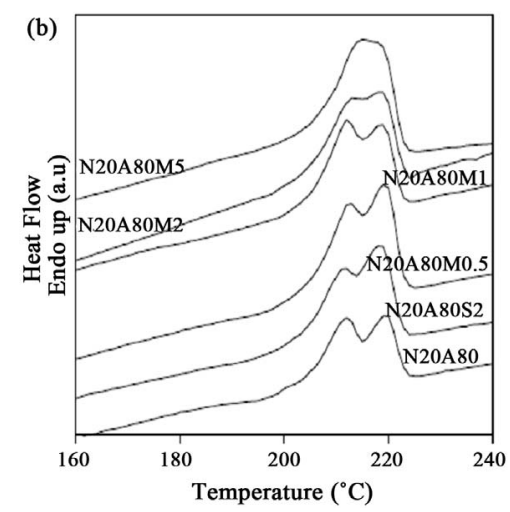

(b)

Figure 18. DSC: (a) cooling and (b) second heating scans of blends of PA6/ABS with and without SMA modified MWNT [11].

g-MA), SMA or dual compatibilizer with MWNTs. They found the good dispersion of MWNTs leading to higher number of heterogeneous nucleating sites available for crystallization of PP in the blend containing polar components viz. PP-g-MA, SMA or dual compatibilizer.

Bose et al. [11] have been reported fractionated crystallization in melt-mixed blend of 20/80 PA6/ABS with or without SMA and SMA modified composites. They observed that SMA and MWNTs content crucially influencing the fractionated crystallization of 20/80 PA6/ABS blends. It is also concluded that MWNTs are acting as a strong heterogeneity (nucleating) center manifested by an increase in the crystallization temperature.

Manchando et al. [36] reported a significant increase in the glass transition temperature $\left(\mathrm{T}_{\mathrm{g}}\right)$ of SWNTs incurporated natural rubber composites as well as SWNTs were found to accelerate the vulcanization reaction.

\subsection{Rheology of Polymer/CNTs Composites}

The type and concentrations of CNTs significantly influences the phase morphology and rheological properties of polymer in the final composite form. Limited understanding of the rheological behavior of the composite materials has been reported. Pötschke et al. [25] thoroughly investigated the rheological behavior of polycarbonate (PC) containing between 0.5 to $15 \mathrm{wt} \%$ CNTs with the help of dynamic oscillatory shear measurement technique at $260^{\circ} \mathrm{C}$. They studied the various masterbatch diluted composites and pure PC on complex viscosity at various frequencies as shown in the Figure 19. An increase in complex viscosity associated with addition of CNTs was found much higher than the changes of viscosity reported for carbon nanofiber having larger diameters for carbon black composites.

Bose et al. [37] also found a significant enhancement of melt viscosity for the blends (PA6/ABS) in the presence of SMA modified MWNTs. It has been also reported that, the presence of MWNTs in the compatibilizer phase led to additional increase in viscosity and storage modulus of the nanocomposites. They concluded that these observed increase in melt viscosity is apparently due to combined effect of reactive compatibilization and changes in the rheological behavior of the interphases by addition of MWNTs.

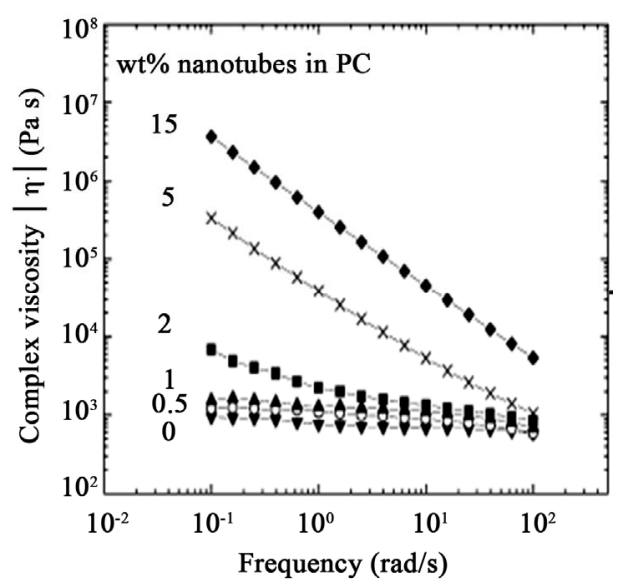

Figure 19. Complex viscosity of CNTs filled polycarbonate [25]. 
In short, CNTs based polymer nanocomposites are the most promising advanced materials for the society if able to achieve the desirable properties by reducing the issues.

\section{REFERENCES}

[1] P. M. Ajayan and J. M. Tour, "Nanotube Composites," Nature, Vol. 447, No. 7148, 2007, pp. 1066-1068. doi:10.1038/4471066a

[2] M. Moniruzzaman and K. I. Winey, "Review Polymer Nanocomposites Containing Carbon Nanotubes," Macromolecules, Vol. 39, No. 16, 2006, pp. 5194-5205. doi: $10.1021 / \mathrm{ma} 060733 \mathrm{p}$

[3] P. M. Ajayan, "Nanotubes from Carbon," Chemical Reviews, Vol. 99, No. 7, 1999, pp. 1787-1799. doi:10.1021/cr970102g

[4] R. H. Baughman, A. A. Zakhidov and W. A. de Heer, "Carbon Nanotubes-The Route toward Applications," Science, Vol. 297, No. 5582, 2002, pp. 787-792. doi:10.1126/science. 1060928

[5] A. M. K. Esawi and M. M. Farag, "Carbon Nanotube Reinforced Composites: Potential and Current Challenges," Materials and Design, Vol. 28, No. 9, 2007, pp. 2394-2401. doi:10.1016/j.matdes.2006.09.022

[6] J. B. Bai and A. Allaoui, "Effect of the Length and the Aggregate Size of MWNTs on the Improvement Efficiency of the Mechanical and Electrical Properties of Nanocomposites-Experimental Investigation," Composites: Part $A$, Vol. 34, No. 8, 2003, pp. 689-694. doi:10.1016/S1359-835X(03)00140-4

[7] S. B. Kharchenko, J. F. Douglas1, J. Obrzut 1, E. A. Grulke and K. Migler, "Flow-Induced Properties of Nanotube-Filled Polymer Materials," Nature Materials, Vol. 3, No. 8, 2004, pp. 564-568. doi:10.1038/nmat1183

[8] H. Pernot, M. Baumert, F. Court and L. Leibler, "Polymer Blends," Nature Materials, Vol. 1, 2002, pp. 54-58. doi:10.1038/nmat 711

[9] Y.-H. Liao, "Manufacturing Process of Nanotube/Nanofiber Nanocomposite: Dispersion and Alignment Study," Ph.D. Thesis, The Florida State University, Fort Lauderdale, 2007.

[10] J. L. Carbon, "Nanotube/Polymer Composites and Novel Micro- and Nano- Structured Electrospun Polymer Materials," Ph.D. Thesis, Georgia Institute of Technology, Atlanta, 2007.

[11] S. Bose, A. R. Bhattacharyya, A. R. Kulkarni and P. Pötschke, "Electrical, rheological and Morphological Studies in Co-Continuous Blends of Polyamide6 and Acrylonitrile-Butadiene-Styrene with Multiwall Carbon Nanotubes Prepared by Melt Blending," Lectrical, Rheological and Morphological Studies in Co-Continuous, Vol. 69, No. 3-4, 2008, pp. 4202-4230.

[12] S. Bose, A. R. Bhattacharyya, L. Häußler and P. Pötschke, "Influence of Multiwall Carbon Nanotubes on the Mechanical Properties and Unusual Crystallization Behaviour in Melt-Mixed Co-Continuous Blends of Polyamide6 and Acrylonitrile-Butadienestyrene," Polymer Engi- neering \& Science, Vol. 49, No. 8, 2008, pp. 3010-3022.

[13] M. Abdel-Goad and P. Potschke, "Rheological Characterization of Melt Processed Polycarbonate-Multiwalled carbon Nanotube Composites," Journal of Non-Newtonian Fluid Mechanics, Vol. 128, No. 1, 2005, pp. 2-6. doi:10.1016/j.jnnfm.2005.01.008

[14] A. R. Bhattacharyyaa, 1, T. V. Sreekumara, T. Liua, S. Kumara, L. M. Ericsonb, R. H. Haugeb and R. E. Smalleyb, "Crystallization and Orientation Studies in Polypropylene/Single Wall Carbon Nanotube Composite," Polymer, Vol. 44, No. 8, 2003, pp. 2373-2377.

[15] P. Pötschke, A. R. Bhattacharyya and A. Janke, "Carbon Nanotube-Filled Polycarbonate Composites Produced by Melt Mixing and Their Use in Blends with Polyethylene," Carbon, Vol. 42, No. 5-6, 2004, pp. 965-969. doi:10.1016/j.carbon.2003.12.001

[16] A. R. Bhattacharya, P. Pötschke, M. Abdel-Goad and D. Fischer, "Effect of Encapsulated SWNT on the Mechanical Properties of Melt Mixed PA12/SWNT Composites," Chemical Physics Letters, Vol. 392, No. 1-3, 2004, pp. 28-37. doi:10.1016/i.cplett.2004.05.045

[17] T. Kashiwagi, J. Fagan, J. F. Douglas and K. Yamamoto, "Relationship between Dispersion Metric and Properties of PMMA/SWNT Nanocomposites," Polymer, Vol. 48, No. 16, 2007, pp. 4855-4866.

[18] Y. Li, H. Shimizu, "Co-Continuous Polyamide 6 (PA6)/ Acrylonitrile-Butadiene-Styrene (ABS) Nanocomposites," Macromolecular Rapid Communications, Vol. 26, No. 9, 2005, pp. 710-715. doi:10.1002/marc.200400654

[19] M. A. L. Manchado, L. Valentini, J. Biagiotti and J. M. Kenny, "Thermal and Mechanical Properties of SingleWalled Carbon Nanotubes-Polypropylene Composites Prepared by Melt Processing," Carbon, Vol. 43, No. 7, 2005, pp. 1499-1505. doi:10.1016/j.carbon.2005.01.031

[20] S. Bose, A. R. Bhattacharyya, P. V. Kodgire and A. Misra, "Fractionated Crystallization in PA6/ABS Blends: Influence of a Reactive Compatibilizer and Multiwall CNT," Polymer, Vol. 48, No. 1, 2007, pp. 356-362. doi:10.1016/i.polymer.2006.11.019

[21] V. J. Triacca, S. Ziaee, J. W. Barlow, H. Keskula and D. R. Paul, "Reactive Compatibilization of Blends of Nylon 6 and ABS Materials," Polymer, Vol. 32, No. 8, 1994, pp. 1401-1413. doi:10.1016/0032-3861(91)90420-N

[22] M. Moniruzzaman, J. Chattopadhyay, W. E. Billups and K. I. Winey, "Tuning the Mechanical Properties of SWNT/ Nylon 6,10 Composites with Flexible Spacers at the Interface," Nano Letters, Vol. 7, No. 5, 2007 pp. 1178-1185. doi:10.1021/n1062868e

[23] P. Liu, "Modifications of carbon nanotubes with Polymers," European Polymer Journal, Vol. 41, No. 11, 2005 pp. 2693-2703. doi:10.1016/j.eurpolymj.2005.05.017

[24] S. Bose, A. R. Bhattacharyya, R. A. Khare, A. R. Kulkarni, T. U. Patro and P. Sivaraman, "Tuning the Dispersion of Multiwall CNTs in Co-Continuous Polymer Blends: A Generic Approach," Nanotechnology, Vol. 19, No. 33, 2008, pp. 3304-3312.

[25] P. Pötschke, A. R. Bhattacharyya and A. Janke, "Morphology and Electrical Resistivity of Melt Mixed Blends 
of Polyethylene and Carbon Nanotube Filled Polycarbonate," Polymer, Vol. 44, No. 26, 2003, pp. 8061-8069. doi:10.1016/i.polymer.2003.10.003

[26] T. Liu, I. Y. Phang, L. Shen, S. Y. Chow and W. D. Zhang, "Morphology and Mechanical Properties of MWNT Reinforced Nylon 6 Composites," Macromolecules, Vol. 37, No. 19, 2004 pp. 7214-7222. doi: $10.1021 / \mathrm{ma} 049132 \mathrm{t}$

[27] J. Sandler, M. S. P. Shaffer, T. Prasse, W. Bauhofer, K. Schulte and A. H. Windle, "Development of a Dispersion Process for Carbon Nanotubes in an Epoxy Matrix and the Resulting Electrical Properties," Polymer, Vol. 40, No. 21, 1999, pp. 5967-5971. doi:10.1016/S0032-3861(99)00166-4

[28] S. Kumar, H. Doshi, M. Srinivasarao, J. O. Park and D. A. Schiraldi, "Fibers from Polypropylene/Nano Carbon Fiber Composites," Polymer, Vol. 43, No. 5, 2002, pp. 1701-1703. doi:10.1016/S0032-3861(01)00744-3

[29] B. Safadi, R. Andrews and E. A. Grulke, "Multiwalled Carbon Nanotube Polymer Composite: Synthesis and Characterization," Journal of Applied Polymer Science, Vol. 84, No. 14, 2002, pp. 2660-2669. doi:10.1002/app.10436

[30] B. F. Jogi, "Structure Property Relationship Studies on Carbon Nanotubes Based Polymer Blends," PhD Thesis, Indian Institute of Technology Bombay, Mumbai, 2012.

[31] O. Meincke, D. Kaempfer, H. Weickmann, C. Friedrich, M. Vathauer and H. Warth, "Mechanical Properties and Electrical Conductivity of Carbon-Nanotube Filled Polyamide-6 and Its Blends with Acrylonitrile/Butadiene/Styrene," Journal of Polymer Science, Vol. 45, No. 3, 2004, pp. 739-748.

[32] Z. X. Jin, K. P. Pramoda and S. H. Goh, "Dynamic Me- chanical Behaviour of Melt Mixed Polymer Nanocomposite," Chemical Physics Letter, Vol. 337, 2001, pp. 41-47.

[33] P. V. Kodgire, A. R. Bhattacharyya, S. Bose, N. Gupta, A. R. Kulkarni and A. Misra, "Control of Multiwall Carbon Nanotubes Dispersion in Polyamide6 Matrix: An Assessment through Electrical Conductivity," Chemical Physics Letters, Vol. 432, No. 4-6, 2006, pp. 480-485. doi:10.1016/j.cplett.2006.10.088

[34] S. B. Jagtap. R. K. Kushwaha and D. Ratna, "Poly(Ethylene Oxide)-Multiwall Carbon Nanotube Composites: Effect of Dicarboxylic Acid Salt-Based Modifiers," Applied Polymer Science, 2012, pp. 38112-38121. doi:10 1002/APP

[35] R. A. Khare, A. R. Bhattacharyya, A. R. Kulkarni, M. Saroop and A. Biswas, "The State of Dispersion of MWNT in PP/ABS Blends: Investigating the Morphological and Electrical Properties," International Conference on Advanced Materials (ICAM 2008), Kottayam, 18-21 February 2008. www.chemistry-conferences.com/2008/02/18 - 21 Conference on $-5 \mathrm{k}$

[36] M. A. L. Manchado, J. Biagiotti, L. Valentini and J. M. Kenny, "Dynamic Mechanical and Raman Spectroscopy Studies on Interaction between Single-Walled Carbon Nanotubes and Natural Rubber," Journal of Applied Polymer Science, Vol. 92, No. 5, 2004, pp. 3394-3400. doi:10.1002/app.20358

[37] S. Bose, A. R. Bhattacharyya, L. Häußler and P. Pötschke, "Influence of Multiwall Carbon Nanotubes on the Mechanical Properties and Unusual Crystallization Behaviour in Melt-Mixed Co-Continuous Blends of Polyamide6 and Acrylonitrile-Butadienestyrene," Polymer Engineering \& Science, Vol. 49, No. 8, 2008, pp. 3010-3022. 